SNS-PH/00-11

IFUP-TH 21/2000

\title{
Localization of Quantum Fields on Branes
}

\author{
Mihail Mintchev \\ Istituto Nazionale di Fisica Nucleare, Sezione di Pisa \\ Dipartimento di Fisica dell'Università di Pisa, \\ Via Buonarroti 2, 56127 Pisa, Italy \\ Luigi Pilo \\ Scuola Normale Superiore, \\ Piazza dei Cavalieri 7, 56126 Pisa, Italy \\ Istituto Nazionale di Fisica Nucleare, Sezione di Pisa
}

\begin{abstract}
A mechanism for localization of quantum fields on a $s$-brane, representing the boundary of a $s+2$ dimensional bulk space, is investigated. Minkowski and AdS bulk spaces are analyzed. Besides the background geometry, the relevant parameters controlling the theory are the mass $M$ and a real parameter $\eta$, specifying the boundary condition on the brane. The importance of exploring the whole range of allowed values for these parameters is emphasized. Stability in Minkowski space requires $\eta \geq-M$, whereas in the AdS background all real $\eta$ are permitted. Both in the flat and in AdS case, the induced field on the brane is a non-canonical generalized free field. For a suitable choice of boundary condition, corresponding to the presence of a boundary state, the induced field on the brane mimics standard $s+1$ dimensional physics. In a certain range of $\eta$, the spectral function i $\mathrm{n}$ the the AdS case is dominated by a massive excitation, which imitates the presence of massive particle on the brane. We show that the quantum field induced on the brane is stable.
\end{abstract}

June 2000 


\section{Introduction}

The scenario of a physical space-time with large extra dimensions is now attracting much interest. According to this proposal [1], our world is confined in a four-dimensional defect, a 3-brane, embedded in a higher dimensional space $\mathcal{M}$, called bulk space. The extra dimensions have sub-millimeter size, which can be tested, in principle, in future collider experiments [2]. From a geometric point of view, the bulk space is a product of the Minkowski space $\mathbf{M}_{3+1}$ with a suitable compact space like in the Kaluza-Klein (KK) program. Further insights come from the Randall-Sundrum solution [3, [4], where $\mathcal{M}$ is a slice of the anti-de Sitter (AdS) space-time in five dimensions. Localization of gravity is realized through the "zero mode" of the five-dimensional gravitational field, which is appreciably different from zero only close to the 3-brane of our world. Despite that the extra fifth dimension is infinite, the localization mechanism works also in the noncompact case [4] and Newton's law is essentially recovered [0]. Another class of models [7, 8], potentially interesting for the cosmological constant problem, is obtained when the bulk space has an additional internal negative-tension brane, which separates an AdS region from a flat region. In this case, the Newtonian potential on the world brane is recovered only at intermediate scales [7, 9], however there are some issues [10, 11, 12, 13] related with unitarity and stability which are still not completely understood.

In this paper we will be concerned with the mechanism responsible for the localization of a quantum scalar field on a brane, representing the boundary of an infinite bulk space. More precisely, we address the following quantum field theory problem. Let $\Phi$ be a local quantum field with prescribed dynamics, propagating in a bulk space $\mathcal{M}$ with a nontrivial boundary $\partial \mathcal{M}$. The problem is to construct and investigate the field $\varphi$ induced by $\Phi$ on $\partial \mathcal{M}$. Referring in what follows to $\varphi$ as the induced field, our main goal is to study the general mechanism allowing to localize a quantum field on $\partial \mathcal{M}$. We also analyze the interplay between locality on $\mathcal{M}$ and $\partial \mathcal{M}$ and the relationship between the mass spectra of $\Phi$ and $\varphi$. Our interest in this sort of quantum boundary value problem is not new [14, 15, 16]. In order to establish a contact with the previous work, we start by considering a flat bulk space which is both instructive and provides a reference model for the study of the more involved AdS background. Our investigation shows that the behavior of $\varphi$ is controlled essentially by two parameters: the bulk mass $M \geq 0$ and a real parameter $\eta$ (with dimension of mass), which specifies a generic mixed boundary condition on $\partial \mathcal{M}$. One of the main differences between the flat and the AdS bulk space concerns the allowed values of $\eta$. In the AdS case, $\eta$ can take any real value, whereas for flat $\mathcal{M}$ the requirement of stability implies $\eta \geq-M$. It turns out that besides the

continuum KK modes, for a suitable value $\eta_{b}(M)$ of $\eta$, the spectrum contains a "zero mode", decaying exponentially out of the brane and generating massless excitations on it. 
This feature, which was already established in the case $M=0$, extends therefore also to $M>0$. A remarkable consequence of this fact is that short range interactions in the flat bulk space $(M>0)$ induce long range interactions on the brane when $\eta=\eta_{b}$. For $M>0$ and $\eta \neq \eta_{b}$ the induced field $\varphi$ has a mass-gap in flat bulk space and no gap in the AdS background. The potential between two static sources on the brane behaves accordingly.

We stress that for establishing these results it is fundamental to have under control the whole range of the parameter $\eta$. Some partial results for $\eta=0$ have been derived recently in [17, 6]. Lifting this restriction is however essential, because it turns out for instance that $\eta_{b} \neq 0$ for $M>0$. The study of the problem for general admissible $\eta$ is therefore crucial and is among the main achievements of this paper. Another interesting aspect, discussed below, is the behavior of the continuum mass spectrum in the AdS case. For generic values of $\eta$ and $M$ the spectral function entering the Källén-Lehmann representation for $\varphi$ is featureless. For certain values of $\eta$ however (for instance when $\eta$ is close to $\eta_{b}(M)$ ), it develops a narrow and high peak, which corresponds to excitations with sharply localized mass.

Concluding this preliminary part, we would like to fix the action for the field $\Phi$. It reads

$$
S=\frac{1}{2} \int_{\mathcal{M}} \sqrt{G} d x^{(s+2)}\left[G^{A B} \partial_{A} \Phi \partial_{B} \Phi-M^{2} \Phi^{2}\right]-\frac{1}{2} \int_{\partial \mathcal{M}} \sqrt{G_{\text {in }}} d x^{(s+1)} \eta \Phi^{2} .
$$

The bulk space $\mathcal{M}$ has a Lorentzian metric $G_{A B}$; its time-like boundary $\partial \mathcal{M}$ (a $s$-brane) inherits the induced metric $G_{\text {in }}$. The variation of $S$ gives both the equation of motion

$$
G^{A B} \nabla_{A} \nabla_{B} \Phi+M^{2} \Phi=0
$$

and the boundary condition

$$
\left.\left(n^{A} \nabla_{A} \Phi-\eta \Phi\right)\right|_{\partial \mathcal{M}}=0, \quad \eta \in \mathbb{R}
$$

where $n^{A}$ is the unit normal of $\partial \mathcal{M}$.

The paper is organized as follows: in the next section we take $G$ to be flat, whereas in section 3 we consider the AdS metric. The last section is devoted to our conclusions.

\section{Flat bulk space}

The purpose of this section is to illustrate, in its simplest form, a general mechanism for inducing quantum fields on a brane. Let us consider the manifold $\mathcal{M}=\mathbb{R}^{s+1} \times \mathbb{R}_{+}$, where $\mathbb{R}_{+}$is the half line $\{y \in \mathbb{R}: y>0\}$. We adopt the coordinates $(x, y) \in \mathbb{R}^{s+1} \times \mathbb{R}_{+}$and the 
notations $x \equiv\left(x^{0}, x^{1}, \ldots, x^{s}\right)=\left(x^{0}, \mathbf{x}\right)$ and $p \equiv\left(p^{0}, p^{1}, \ldots, p^{s}\right)=\left(p^{0}, \mathbf{p}\right)$. Our first task will be to analyze the free scalar quantum field $\Phi(x, y)$, which propagates on $\mathcal{M}=\mathbb{R}^{s+1} \times \mathbb{R}_{+}$ equipped with the flat metric

$$
G_{A B}=\left(\begin{array}{cc}
g & 0 \\
0 & -1
\end{array}\right), \quad \operatorname{diag} g=(1,-1, \ldots,-1) .
$$

The boundary $\partial M$ coincides with the $s+1$-dimensional Minkowski space $\mathbf{M}_{s+1} \equiv\left\{\mathbb{R}^{s+1}, g\right\}$ and represents a $s$-brane. Eq. (2) gives rise to

$$
\left(\square_{G}+M^{2}\right) \Phi(x, y)=0,
$$

where $\square_{G}$ is the Laplacian associated with (雨) and $M \geq 0$ is the mass. Let us introduce

$$
K \equiv-\sum_{i=1}^{s} \partial_{i}^{2}-\partial_{y}^{2}+M^{2}
$$

Then

$$
\square_{G}+M^{2}=\partial_{0}^{2}+K
$$

and the quantization of Eq. (5) requires the study of the operator $K$. In order to apply the standard and well known procedure, one needs a self-adjoint positive $K$. The subtle point in analyzing $K$ concerns the term $-\partial_{y}^{2}$, which is defined on the half line $\mathbb{R}_{+}$. We recall [19] in this respect that $\mathrm{K}$ is not self-adjoint but only Hermitian in the space $C_{0}^{\infty}\left(\mathbb{R}^{s} \times \mathbb{R}_{+}\right)$of infinitely differentiable functions with compact support. The relative deficiency indices are however equal, because $K$ commutes with the complex conjugation. Therefore, $K$ admits self-adjoint extensions. All of them are parametrized by a real parameter $\eta$ and correspond to the boundary condition

$$
\lim _{y \downarrow 0}\left(\partial_{y}-\eta\right) \Phi(x, y)=0,
$$

which is just eq.(3) applied to the case we are considering. The parameter $\eta$ has dimension of mass. Eq.(\$) represents the so called mixed boundary condition (in the limit $\eta \rightarrow$ 0 and $\eta \rightarrow \infty$ one recovers the familiar Neumann and Dirichlet boundary conditions respectively). The associated self-adjoint operator will be denoted by $K_{\eta}$.

At this point, the main step in constructing the quantum field $\Phi$ is to identify a complete orthonormal set of eigenfunctions of $K_{\eta}$. For this purpose we first focus on the operator $-\partial_{y}^{2}$ and consider the family of functions

$$
\mathcal{B}= \begin{cases}\left\{\psi(y, \lambda): y, \lambda \in \mathbb{R}_{+}\right\} & \text {if } \eta \geq 0 \\ \left\{\psi(y, \lambda), \psi_{b}(y): y, \lambda \in \mathbb{R}_{+}\right\} & \text {if } \eta<0\end{cases}
$$


where

$$
\psi(y, \lambda)=\mathrm{e}^{-i \lambda y}+B(\lambda) \mathrm{e}^{i \lambda y}, \quad B(\lambda)=\frac{\lambda-i \eta}{\lambda+i \eta}
$$

and

$$
\psi_{b}(y)=\sqrt{2|\eta|} \mathrm{e}^{\eta y}
$$

The elements of $\mathcal{B}$ satisfy the boundary condition

$$
\lim _{y \downarrow 0}\left(\partial_{y}-\eta\right) \psi(y)=0
$$

and have transparent physical interpretation in the quantum mechanical problem defined by the Hamiltonian $-\partial_{y}^{2}$ on $\mathbb{R}_{+}$: the functions $\psi(y, \lambda)$ represent scattering states $(B(\lambda)$ is the reflection coefficient from the boundary at $y=0$ ), whereas $\psi_{b}$ describes the bound state (with energy $-\eta^{2}$ ), present for $\eta<0$. We will refer in what follows to $\psi_{b}$ as boundary state and we will show that it gives an essential contribution to the quantum field induced on the boundary $\mathbf{M}_{s+1}$.

One can verify the following orthogonality and completeness relations:

$$
\begin{gathered}
\int_{0}^{\infty} d y \bar{\psi}\left(y, \lambda_{1}\right) \psi\left(y, \lambda_{2}\right)=2 \pi \delta\left(\lambda_{1}-\lambda_{2}\right), \quad \lambda_{1}, \lambda_{2} \in \mathbb{R}_{+}, \\
\int_{0}^{\infty} d y \bar{\psi}(y, \lambda) \psi_{b}(y)=0, \quad \lambda \in \mathbb{R}_{+}, \quad \eta<0, \\
\int_{0}^{\infty} \frac{d \lambda}{2 \pi} \bar{\psi}\left(y_{1}, \lambda\right) \psi\left(y_{2}, \lambda\right)+\theta(-\eta) \psi_{b}\left(y_{1}\right) \psi_{b}\left(y_{2}\right)=\delta\left(y_{1}-y_{2}\right), \quad y_{1}, y_{2} \in \mathbb{R}_{+},
\end{gathered}
$$

where

$$
\theta(\alpha)= \begin{cases}1 & \text { if } \alpha>0 \\ 0 & \text { if } \alpha \leq 0\end{cases}
$$

Therefore, $\mathcal{B}$ is a complete orthonormal set of eigenfunctions for the operator $-\partial_{y}^{2}$. Accordingly,

$$
\mathcal{B}\left(K_{\eta}\right)= \begin{cases}\left\{\mathrm{e}^{-i \mathbf{p x}} \psi(y, \lambda): y, \lambda \in \mathbb{R}_{+}, \mathbf{x}, \mathbf{p} \in \mathbb{R}^{s}\right\} & \text { if } \eta \geq 0 \\ \left\{\mathrm{e}^{-i \mathbf{p x}} \psi(y, \lambda), \mathrm{e}^{-i \mathbf{p x}} \psi_{b}(y): y, \lambda \in \mathbb{R}_{+}, \mathbf{x}, \mathbf{p} \in \mathbb{R}^{s}\right\} & \text { if } \eta<0\end{cases}
$$


is such a set for the operator $K_{\eta}$. For the spectrum one has

$$
\mathcal{S}\left(K_{\eta}\right)= \begin{cases}\left\{\kappa \in \mathbb{R}: \kappa \geq M^{2}\right\} & \text { if } \eta \geq 0, \\ \left\{\kappa \in \mathbb{R}: \kappa \geq M^{2}-\eta^{2}\right\} & \text { if } \eta<0 .\end{cases}
$$

Therefore, $K_{\eta}$ is positive for any $\eta \geq 0$. In the range $\eta<0$, the requirement of positivity implies

$$
-M \leq \eta<0
$$

In order to avoid imaginary energies for the field $\Phi$, in what follows we impose (19). Notice that for $M=0$, this condition excludes negative values of $\eta$. We shall see in the next section that this is not the case for the AdS space, which is essential in the Randall-Sundrum scenario.

Now, we are in position to construct the local quantum field $\Phi$. The content of $\mathcal{B}\left(K_{\eta}\right)$ suggest the presence of two building blocks:

$$
\begin{gathered}
\phi(x, y)=\int_{-\infty}^{\infty} \frac{d^{s} p}{(2 \pi)^{s}} \int_{0}^{\infty} \frac{d \lambda}{2 \pi} \frac{1}{\sqrt{2 \omega_{M^{2}+\lambda^{2}}(\mathbf{p})}} \cdot \\
{\left[a^{*}(\mathbf{p}, \lambda) \mathrm{e}^{i \omega_{M^{2}+\lambda^{2}}(\mathbf{p}) x^{0}-i \mathbf{x p}} \psi(y, \lambda)+a(\mathbf{p}, \lambda) \mathrm{e}^{-i \omega_{M^{2}+\lambda^{2}}(\mathbf{p}) x^{0}+i \mathbf{p x}} \bar{\psi}(y, \lambda)\right], \quad-M \leq \eta,}
\end{gathered}
$$

and

$$
\begin{gathered}
\chi(x, y)=\int_{-\infty}^{\infty} \frac{d^{s} p}{(2 \pi)^{s}} \frac{1}{\sqrt{2 \omega_{M^{2}-\eta^{2}}(\mathbf{p})}} \cdot \\
{\left[b^{*}(\mathbf{p}) \mathrm{e}^{i \omega_{M^{2}-\eta^{2}}(\mathbf{p}) x^{0}-i \mathbf{x p}} \psi_{b}(y)+b(\mathbf{p}) \mathrm{e}^{-i \omega_{M^{2}-\eta^{2}}(\mathbf{p}) x^{0}+i \mathbf{p x}} \bar{\psi}_{b}(y)\right], \quad-M \leq \eta<0,}
\end{gathered}
$$

where

$$
\omega_{m^{2}}(\mathbf{p})=\sqrt{\mathbf{p}^{2}+m^{2}}
$$

We assume also that $\left\{a^{*}(\mathbf{p}, \lambda), a(\mathbf{p}, \lambda)\right\}$ commute with $\left\{b^{*}(\mathbf{p}), b(\mathbf{p})\right\}$ and satisfy the canonical commutation relations:

$$
\begin{gathered}
{\left[a\left(\mathbf{p}_{1}, \lambda_{1}\right), a^{*}\left(\mathbf{p}_{2}, \lambda_{2}\right)\right]=(2 \pi)^{s+1} \delta\left(\mathbf{p}_{1}-\mathbf{p}_{2}\right) \delta\left(\lambda_{1}-\lambda_{2}\right),} \\
{\left[a\left(\mathbf{p}_{1}, \lambda_{1}\right), a\left(\mathbf{p}_{2}, \lambda_{2}\right)\right]=\left[a^{*}\left(\mathbf{p}_{1}, \lambda_{1}\right), a^{*}\left(\mathbf{p}_{2}, \lambda_{2}\right)\right]=0,} \\
{\left[b\left(\mathbf{p}_{1}\right), b^{*}\left(\mathbf{p}_{2}\right)\right]=(2 \pi)^{s} \delta\left(\mathbf{p}_{1}-\mathbf{p}_{2}\right),} \\
{\left[b\left(\mathbf{p}_{1}\right), b\left(\mathbf{p}_{2}\right)\right]=\left[b^{*}\left(\mathbf{p}_{1}\right), b^{*}\left(\mathbf{p}_{2}\right)\right]=0 .}
\end{gathered}
$$


Let us summarize the basic properties of the fields (20,21). By construction both $\phi$ and $\chi$ satisfy Eqs. (5, 8). At this point, the requirement of local commutativity plays a crucial role. Indeed, one can verify that:

- (i) $\phi$ is local for $\eta \geq 0$;

- (ii) neither $\phi$ nor $\chi$ are local for $-M \leq \eta<0$, however their sum $\phi+\chi$ is local.

Therefore, the local field in $\mathbb{R}^{s+1} \times \mathbb{R}_{+}$we are looking for, reads

$$
\Phi(x, y)= \begin{cases}\phi(x, y) & \text { if } \eta \geq 0 \\ \phi(x, y)+\chi(x, y) & \text { if }-M \leq \eta<0\end{cases}
$$

The completeness of the system $\mathcal{B}\left(K_{\eta}\right)$ implies that that above defined $\Phi$ satisfies also the canonical commutation relation

$$
\left[\left(\partial_{0} \Phi\right)\left(x^{0}, \mathbf{x}_{1}, y_{1}\right), \Phi\left(x^{0}, \mathbf{x}_{2}, y_{2}\right)\right]=-i \delta\left(\mathbf{x}_{1}-\mathbf{x}_{2}\right) \delta\left(y_{1}-y_{2}\right) .
$$

The two-point vacuum expectation values (Wightman functions) of the fields $\phi$ and $\chi$ in the Fock representation of the algebra (23,24) are easily derived:

$$
\left\langle\phi\left(x_{1}, y_{1}\right) \phi\left(x_{2}, y_{2}\right)\right\rangle_{0}=\int_{0}^{\infty} \frac{d \lambda}{2 \pi} \bar{\psi}\left(y_{1}, \lambda\right) \psi\left(y_{2}, \lambda\right) W_{M^{2}+\lambda^{2}}\left(x_{12}\right),
$$

and

$$
\left\langle\chi\left(x_{1}, y_{1}\right) \chi\left(x_{2}, y_{2}\right)\right\rangle_{0}=\bar{\psi}_{b}\left(y_{1}\right) \psi_{b}\left(y_{2}\right) W_{M^{2}-\eta^{2}}\left(x_{12}\right)
$$

where $x_{12} \equiv x_{1}-x_{2}$ and

$$
W_{m^{2}}(x)=\int_{-\infty}^{\infty} \frac{d^{s} p}{(2 \pi)^{s}} \frac{1}{2 \omega_{m^{2}}(\mathbf{p})} \mathrm{e}^{-i \omega_{m^{2}}(\mathbf{p}) x^{0}+i \mathbf{p x}}=\int_{-\infty}^{\infty} \frac{d^{(s+1)} p}{(2 \pi)^{(s+1)}} \mathrm{e}^{-i p x} \theta\left(p^{0}\right) 2 \pi \delta\left(p^{2}-m^{2}\right)
$$

is the two-point scalar function of mass $m^{2}$ in $\mathbf{M}_{s+1}$. Combining Eqs.(10, 11, 25, 27, 28), one gets

$$
\begin{gathered}
\left\langle\Phi\left(x_{1}, y_{1}\right) \Phi\left(x_{2}, y_{2}\right)\right\rangle_{0}= \\
\int_{0}^{\infty} \frac{d \lambda}{2 \pi} 2\left(\cos \lambda y_{12}+\frac{\lambda^{2}-\eta^{2}}{\lambda^{2}+\eta^{2}} \cos \lambda \widetilde{y}_{12}+\frac{2 \lambda \eta}{\lambda^{2}+\eta^{2}} \sin \lambda \widetilde{y}_{12}\right) W_{M^{2}+\lambda^{2}}\left(x_{12}\right)+ \\
2 \theta(-\eta)|\eta| \mathrm{e}^{\eta \widetilde{y}_{12}} W_{M^{2}-\eta^{2}}\left(x_{12}\right)
\end{gathered}
$$


where $\widetilde{y}_{12} \equiv y_{1}+y_{2}$. Since $\Phi$ is free, its $n$-point correlators are expressed in a standard way in terms of (30), which completes the construction of the local bulk field $\Phi$, satisfying Eqs. (5,8).

So, we are left with the problem of determining the field $\varphi$ induced by $\Phi$ on the boundary $\mathbf{M}_{s+1}$. For this purpose we consider

$$
\left\langle\varphi\left(x_{1}\right) \cdots \varphi\left(x_{n}\right)\right\rangle_{0}=\lim _{y_{i} \downarrow 0}\left\langle\Phi\left(x_{1}, y_{1}\right) \cdots \Phi\left(x_{n}, y_{n}\right)\right\rangle_{0}
$$

It follows from Eq. (30) that $\left\langle\varphi\left(x_{1}\right) \cdots \varphi\left(x_{n}\right)\right\rangle_{0}$ are well defined correlators, which satisfy all standard requirements (Poincaré covariance, local commutativity, positivity and the spectral condition) and therefore uniquely determine, via the reconstruction theorem [20], the induced field $\varphi$. The basic features of $\varphi$ are encoded in the two-point function

$$
\begin{aligned}
& G_{W}\left(x_{12}\right)=\left\langle\varphi\left(x_{1}\right) \varphi\left(x_{2}\right)\right\rangle_{0}=\lim _{y_{i} \downarrow 0}\left\langle\Phi\left(x_{1}, y_{1}\right) \Phi\left(x_{2}, y_{2}\right)\right\rangle_{0}= \\
& \int_{0}^{\infty} \frac{d \lambda}{2 \pi} \frac{4 \lambda^{2}}{\lambda^{2}+\eta^{2}} W_{M^{2}+\lambda^{2}}\left(x_{12}\right)+2 \theta(-\eta)|\eta| W_{M^{2}-\eta^{2}}\left(x_{12}\right) .
\end{aligned}
$$

Notice that $G_{W}$ vanishes in the Dirichlet case $(|\eta|=\infty)$, as it should be. Changing variables, Eq. (32) gives the following Källén-Lehmann representation

$$
G_{W}\left(x_{12}\right)=\int_{0}^{\infty} d \lambda^{2} \varrho\left(\lambda^{2}\right) W_{\lambda^{2}}\left(x_{12}\right)+2 \theta(-\eta)|\eta| W_{M^{2}-\eta^{2}}\left(x_{12}\right),
$$

where

$$
\varrho\left(\lambda^{2}\right)=\theta\left(\lambda^{2}-M^{2}\right) \frac{\sqrt{\lambda^{2}-M^{2}}}{\pi\left(\lambda^{2}+\eta^{2}-M^{2}\right)} .
$$

Obviously $\varphi$ does not satisfy the free Klein-Gordon equation. Since $\varrho$ defines a polynomialy bounded positive measure on $[0,+\infty)$, from Eq. (33) we deduce that $\varphi$ is a generalized free field [20]. We stress that in contrast to the bulk field $\Phi$, the induced field $\varphi$ is not a canonical one. In this sense $\varphi$ cannot be derived from a local Lagrangian on the brane.

The evaluation of the integral in Eq. (33) is straightforward in momentum space. Performing a Fourier transformation, one finds

$$
\widehat{G}_{W}(p)=2 \theta\left(p^{0}\right)\left[\theta\left(p^{2}-M^{2}\right) \frac{\sqrt{p^{2}-M^{2}}}{p^{2}-M^{2}+\eta^{2}}+\theta(-\eta)|\eta| 2 \pi \delta\left(p^{2}-M^{2}+\eta^{2}\right)\right] .
$$

Eq. (35) provides a complete information about the mass spectrum of $\varphi$. For $\eta \geq 0$ the spectrum is continuous and bounded from below by $M$, thus exhibiting a mass gap when 
$M>0$. Such a behavior is expected on general grounds. In the range $-M \leq \eta<0$ the mass spectrum has in addition a discrete point-like contribution, stemming from the boundary state (111). The representation of the Poincare group in this range is a superposition of a continuum mass representation defined by $\varrho$ and a standard particle representation of mass $M^{2}-\eta^{2}$. The particular case

$$
\eta=\eta_{b} \equiv-M,
$$

deserves special attention. In spite of the fact that only short range interactions $(M \neq 0)$ are present in the bulk space $\mathbb{R}^{s+1} \times \mathbb{R}_{+}$, a long range interaction $\left(M^{2}-\eta^{2}=0\right)$ is induced on its boundary $\mathbf{M}_{s+1}$. The physical origin of the remarkable behavior when $-M \leq \eta<0$ is a sort of attraction of the bulk field by the boundary. We expect such boundary interactions to be universal and therefore essential in brane physics in general. In fact, we will show in the next section that the above phenomena take place also in AdS background, thus being relevant for the Randall-Sundrum framework as well.

It is instructive to derive also the propagator of the induced field. From Eq. (33) one obtains

$$
G_{F}\left(x_{12}\right)=i\left\langle T \varphi\left(x_{1}\right) \varphi\left(x_{2}\right)\right\rangle_{0}=\int_{0}^{\infty} d \lambda^{2} \varrho\left(\lambda^{2}\right) \Delta_{\lambda^{2}}\left(x_{12}\right)+2 \theta(-\eta)|\eta| \Delta_{M^{2}-\eta^{2}}\left(x_{12}\right),
$$

where $T$ indicates time ordering and

$$
\Delta_{m^{2}}(x)=-\int_{-\infty}^{\infty} \frac{d^{(s+1)} p}{(2 \pi)^{(s+1)}} \frac{\mathrm{e}^{-i p x}}{p^{2}-m^{2}+i \varepsilon} .
$$

is the well known scalar propagator. The Fourier transform of $G_{F}$ reads

$$
\widehat{G}_{F}(p)=-\frac{\sqrt{M^{2}-p^{2}}-\eta}{p^{2}-M^{2}+\eta^{2}+i \epsilon},
$$

and is convenient for perturbative calculations on the brane.

Let us determine finally the potential $V(r)$ between two static sources on the brane at distance $r=|\mathbf{x}|$. For $s=3$ one finds

$$
V(r)=\int_{-\infty}^{\infty} d x^{0} G_{F}\left(x^{0}, \mathbf{x}\right)=2 \int_{M}^{\infty} d \lambda \lambda \varrho\left(\lambda^{2}\right) \frac{e^{-\lambda r}}{4 \pi r}+2 \theta(-\eta)|\eta| \frac{e^{-r \sqrt{M^{2}-\eta^{2}}}}{4 \pi r} .
$$

Combining Eqs. (34,40), one easily gets the estimate

$$
V(r) \leq \sup _{\lambda \geq M}[\varrho(\lambda)] \frac{e^{-M r}}{4 \pi r^{2}}+2 \theta(-\eta)|\eta| \frac{e^{-r \sqrt{M^{2}-\eta^{2}}}}{4 \pi r} .
$$


One can further study the dependence of $\sup _{\lambda \geq M}[\varrho(\lambda)]$ on $M$ and $\eta$.

This concludes our investigation of the flat bulk space, which provides useful intuition for the AdS case.

\section{Anti-de Sitter bulk space}

We keep below the bulk manifold $\mathcal{M}=\mathbb{R}^{s+1} \times \mathbb{R}_{+}$, but now equipped with the anti-de Sitter (AdS) metric

$$
d s^{2}=G_{A B} d x^{A} d x^{B}=\mathrm{e}^{-2 a y} g_{\mu \nu} d x^{\mu} d x^{\nu}-d y^{2}, \quad a>0,
$$

with a cosmological constant $\Lambda=-6 a^{2}$. The boundary $\partial \mathcal{M}$ still coincides with $\mathbf{M}_{s+1}$ and the problem is to construct and investigate the scalar quantum field $\Phi$, satisfying Eq. (5) with the metric (42) and the boundary condition (8). Following the procedure developed in the previous section, we have to study the operator

$$
K=-\sum_{i=1}^{s} \partial_{i}^{2}-\mathrm{e}^{(s-1) a y} \partial_{y} \mathrm{e}^{-(s+1) a y} \partial_{y}+\mathrm{e}^{-2 a y} M^{2} .
$$

As in the flat case, the main point is to solve

$$
\left[-\mathrm{e}^{(s-1) a y} \partial_{y} \mathrm{e}^{-(s+1) a y} \partial_{y}+\mathrm{e}^{-2 a y} M^{2}\right] \psi(y, \lambda)=\lambda^{2} \psi(y, \lambda),
$$

with the boundary condition (12). Eqs. (44, 12]) give rise to a well studied [21], [22] singular boundary value problem, related to Bessel's equation. It is worth stressing that there is no freedom to impose any boundary condition at $y=\infty$, which is the singular point of the problem. Setting

$$
\nu=\sqrt{\frac{(s+1)^{2}}{4}+\frac{M^{2}}{a^{2}}}, \quad \eta_{b}=\frac{(s+1-2 \nu)}{2} a,
$$

the complete orthonormal set of eigenfunctions for $s \geq 3$ reads

$$
\mathcal{B}= \begin{cases}\left\{\psi(y, \lambda): y, \lambda \in \mathbb{R}_{+}\right\} & \text {if } \eta \neq \eta_{b} \\ \left\{\psi(y, \lambda), \psi_{b}(y): y, \lambda \in \mathbb{R}_{+}\right\} & \text {if } \eta=\eta_{b}\end{cases}
$$

where

$$
\psi(y, \lambda)=\mathrm{e}^{\frac{(s+1)}{2} a y}\left[J_{\nu}\left(\lambda a^{-1} \mathrm{e}^{a y}\right) \widetilde{Y}_{\nu}\left(\lambda a^{-1}\right)-Y_{\nu}\left(\lambda a^{-1} \mathrm{e}^{a y}\right) \widetilde{J}_{\nu}\left(\lambda a^{-1}\right)\right]
$$




$$
\widetilde{Z}_{\nu}(\zeta)=\frac{1}{2 \sqrt{1+\widetilde{\eta}^{2}}}\left[(1-2 \widetilde{\eta}) Z_{\nu}(\zeta)+2 \zeta Z_{\nu}^{\prime}(\zeta)\right], \quad \widetilde{\eta}=\frac{\eta}{a}-\frac{s}{2}
$$

$Z_{\nu}$ denoting the Bessel function $J_{\nu}$ or $Y_{\nu}$ of first and second kind respectively. Finally, the boundary state

$$
\psi_{b}(y)=\sqrt{2 a(\nu-1)} \mathrm{e}^{\eta_{b} y}
$$

is in this case a zero mode of Eq. (44). The completeness relation of the system (46) is expressed by

$$
\int_{0}^{\infty} d \lambda \sigma(\lambda) \bar{\psi}\left(y_{1}, \lambda\right) \psi\left(y_{2}, \lambda\right)+\delta_{\eta \eta_{b}} \bar{\psi}_{b}\left(y_{1}\right) \psi_{b}\left(y_{2}\right)=\frac{1}{\mu\left(y_{1}\right)} \delta\left(y_{12}\right),
$$

where $\mu$ and $\sigma$ are the following measures

$$
\mu(y)=\mathrm{e}^{(1-s) a y}, \quad \sigma(\lambda)=\frac{\lambda a^{-1}}{\widetilde{J}_{\nu}^{2}\left(\lambda a^{-1}\right)+\widetilde{Y}_{\nu}^{2}\left(\lambda a^{-1}\right)},
$$

on $[0, \infty)$. Moreover, one has also the orthogonality relations

$$
\begin{gathered}
\int_{0}^{\infty} d y \mu(y) \bar{\psi}\left(y, \lambda_{1}\right) \psi\left(y, \lambda_{2}\right)=\frac{1}{\sigma\left(\lambda_{1}\right)} \delta\left(\lambda_{12}\right), \quad \lambda_{1}, \lambda_{2} \in \mathbb{R}_{+} \\
\int_{0}^{\infty} d y \mu(y) \bar{\psi}(y, \lambda) \psi_{b}(y)=0, \quad \lambda \in \mathbb{R}_{+} .
\end{gathered}
$$

The above analysis implies that

$$
\mathcal{B}\left(K_{\eta}\right)= \begin{cases}\left\{\mathrm{e}^{-i \mathbf{p x}} \psi(y, \lambda): y, \lambda \in \mathbb{R}_{+}, \mathbf{x}, \mathbf{p} \in \mathbb{R}^{s}\right\} & \text { if } \eta \neq \eta_{b} \\ \left\{\mathrm{e}^{-i \mathbf{p x}} \psi(y, \lambda), \mathrm{e}^{-i \mathbf{p x}} \psi_{b}(y): y, \lambda \in \mathbb{R}_{+}, \mathbf{x}, \mathbf{p} \in \mathbb{R}^{s}\right\} & \text { if } \eta=\eta_{b}\end{cases}
$$

is a complete orthonormal set for the self-adjoint extension $K_{\eta}$ of the operator (43). For the spectrum one has

$$
\mathcal{S}\left(K_{\eta}\right)= \begin{cases}\{\kappa \in \mathbb{R}: \kappa>0\} & \text { if } \eta \neq \eta_{b} \\ \{\kappa \in \mathbb{R}: \kappa \geq 0\} & \text { if } \eta=\eta_{b}\end{cases}
$$

showing that $K_{\eta}$ is positive for any $\eta \in \mathbb{R}$. Comparing to the flat case, we see that the AdS background has an interesting feature: the energy spectrum of the field $\Phi$ is real and positive for any $\eta \in \mathbb{R}$. 
It follows from Eq. (54) that $\Phi$ is given by the superposition

$$
\Phi(x, y)=\phi(x, y)+\delta_{\eta \eta_{b}} \chi(x, y)
$$

where

$$
\begin{gathered}
\phi(x, y)=\int_{-\infty}^{\infty} \frac{d^{s} p}{(2 \pi)^{s}} \int_{0}^{\infty} d \lambda \sigma(\lambda) \frac{1}{\sqrt{2 \omega_{\lambda^{2}}(\mathbf{p})}} \cdot \\
{\left[a^{*}(\mathbf{p}, \lambda) \mathrm{e}^{i \omega_{\lambda^{2}}(\mathbf{p}) x^{0}-i \mathbf{x p}} \psi(y, \lambda)+a(\mathbf{p}, \lambda) \mathrm{e}^{-i \omega_{\lambda^{2}}(\mathbf{p}) x^{0}+i \mathbf{p x}} \bar{\psi}(y, \lambda)\right],} \\
\chi(x, y)=\int_{-\infty}^{\infty} \frac{d^{s} p}{(2 \pi)^{s}} \frac{1}{\sqrt{2 \omega_{0}(\mathbf{p})}} \cdot \\
{\left[b^{*}(\mathbf{p}) \mathrm{e}^{i \omega_{0}(\mathbf{p}) x^{0}-i \mathbf{x p}} \psi_{b}(y)+b(\mathbf{p}) \mathrm{e}^{\left.-i \omega_{0}(\mathbf{p}) x^{0}+i \mathbf{p x} \bar{\psi}_{b}(y)\right] .}\right.}
\end{gathered}
$$

Here $\left\{a^{*}(\mathbf{p}, \lambda), a(\mathbf{p}, \lambda)\right\}$ and $\left\{b^{*}(\mathbf{p}), b(\mathbf{p})\right\}$ satisfy the commutation relations (23,24) with the substitution

$$
2 \pi \delta\left(\lambda_{1}-\lambda_{2}\right) \longmapsto \frac{1}{\sigma\left(\lambda_{1}\right)} \delta\left(\lambda_{1}-\lambda_{2}\right)
$$

in (23). Using Eq. (50) one easily verifies that $\Phi$ satisfies Eq. (26), being therefore a canonical field. Its two point vacuum expectation value admits the representation

$$
\begin{gathered}
\left\langle\Phi\left(x_{1}, y_{1}\right) \Phi\left(x_{2}, y_{2}\right)\right\rangle_{0}= \\
\int_{0}^{\infty} d \lambda \sigma(\lambda) \bar{\psi}\left(y_{1}, \lambda\right) \psi\left(y_{2}, \lambda\right) W_{\lambda^{2}}\left(x_{12}\right)+\delta_{\eta \eta_{b}} \bar{\psi}_{b}\left(y_{1}\right) \psi_{b}\left(y_{2}\right) W_{0}\left(x_{12}\right) .
\end{gathered}
$$

From Eq. (60) one gets

$$
\begin{aligned}
& G_{W}\left(x_{12}\right)=\left\langle\varphi\left(x_{1}\right) \varphi\left(x_{2}\right)\right\rangle_{0}=\lim _{y_{i} \downarrow 0}\left\langle\Phi\left(x_{1}, y_{1}\right) \Phi\left(x_{2}, y_{2}\right)\right\rangle_{0}= \\
& \int_{0}^{\infty} d \lambda \sigma(\lambda) \bar{\psi}(0, \lambda) \psi(0, \lambda) W_{\lambda^{2}}\left(x_{12}\right)+\delta_{\eta \eta_{b}} 2 a(\nu-1) W_{0}\left(x_{12}\right),
\end{aligned}
$$

which is the Källén-Lehmann representation for the induced field $\varphi$ in the AdS case. It follows from Eqs. (47,48) that $G_{W}$ vanishes for $|\eta|=\infty$ (Dirichlet boundary condition). We therefore consider from now on only finite $\eta$. Using some simple identities among Bessel functions, one finds

$$
G_{W}\left(x_{12}\right)=\int_{0}^{\infty} d \lambda \tau(\lambda) W_{\lambda^{2}}\left(x_{12}\right)+\delta_{\eta \eta_{b}} 2 a(\nu-1) W_{0}\left(x_{12}\right)
$$


with

$\tau(\lambda)=\frac{16}{\pi^{2}} \frac{\lambda a^{-1}}{\left[(1-2 \widetilde{\eta}) J_{\nu}\left(\lambda a^{-1}\right)+2 \lambda a^{-1} J_{\nu}^{\prime}\left(\lambda a^{-1}\right)\right]^{2}+\left[(1-2 \widetilde{\eta}) Y_{\nu}\left(\lambda a^{-1}\right)+2 \lambda a^{-1} Y_{\nu}^{\prime}\left(\lambda a^{-1}\right)\right]^{2}}$.

The $\lambda$-integral in Eq. (62) is easily performed in momentum space, giving the following result

$$
\widehat{G}_{W}(p)=2 \pi \theta\left(p^{0}\right)\left[\frac{\theta\left(p^{2}\right)}{2 \sqrt{p^{2}}} \tau\left(\sqrt{p^{2}}\right)+\delta_{\eta \eta_{b}} 2 a(\nu-1) \delta\left(p^{2}\right)\right] .
$$

Like in the flat space, in spite of the fact that $\Phi$ is a canonical field, $\varphi$ is not. There is no mass gap in the spectrum, which contains a zero-mass point-like contribution only for the specific boundary condition $\eta=\eta_{b}$, when the boundary state (49) belongs to $\mathcal{B}\left(K_{\eta}\right)$.

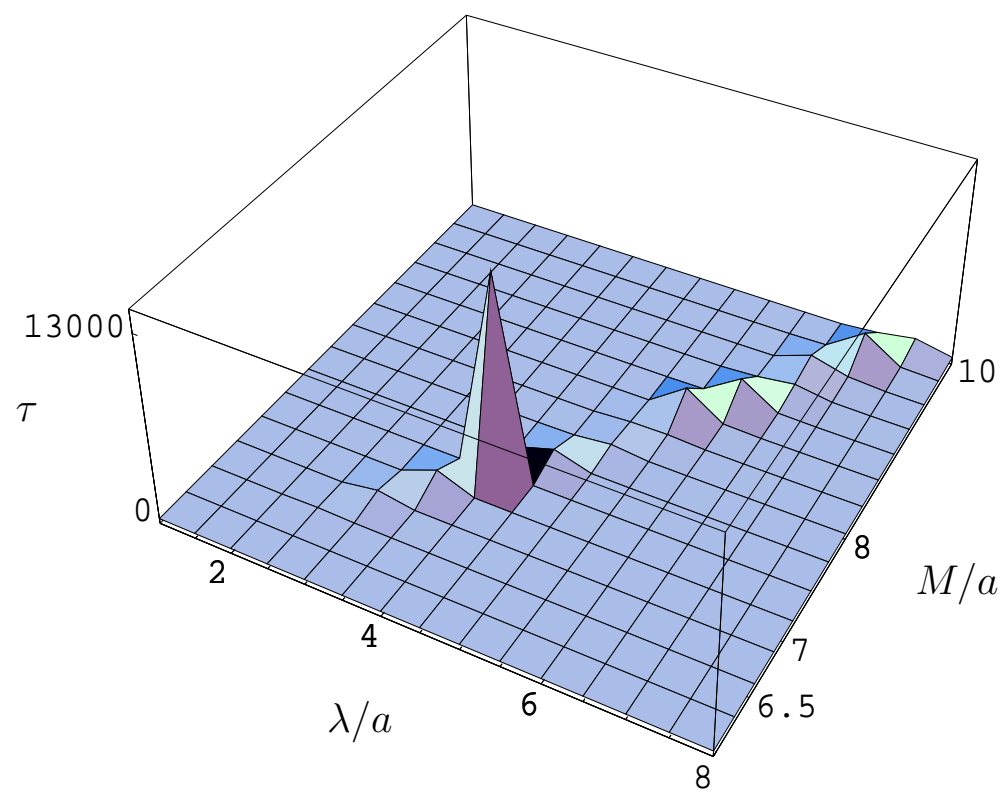

Figure 1. The spectral function $\tau$ for $\eta=\eta_{b}(6 a)$.

It is hard to study analytically the behavior of the measure $\tau$ as a function of $\lambda, M$ and $\eta$. The numerical analysis summarized in Figures 1 and 2 reveals a very interesting 


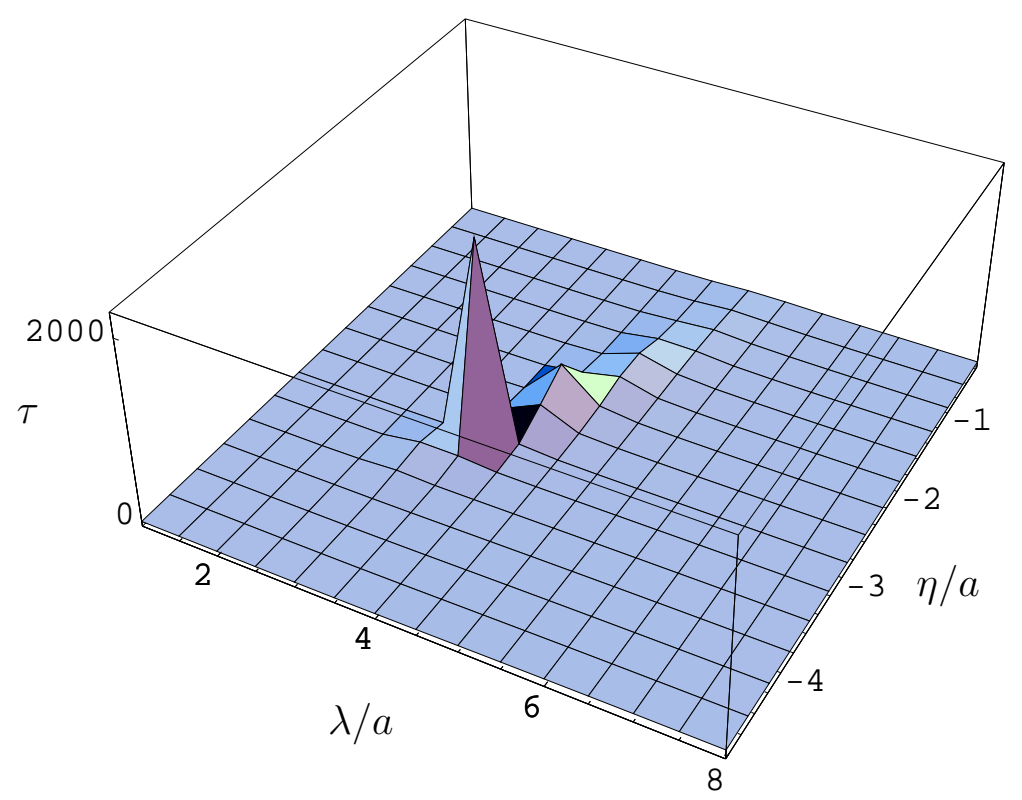

Figure 2. The spectral function $\tau$ for $M=6 a$.

structure. For generic values of $M$ and $\eta$ the spectral function has monotonic behavior. However, when these parameters are close to the curve $\eta=\eta_{b}(M)$ in the plane $(\eta, M)$, a high peak appears in $\tau$, the rest of the spectrum being depressed. As a results a partial localization of a massive mode occurs on the brane. Some doubts have been raised [17] about the stability of the corresponding excitations. In order to clarify the issue, it is necessary to consider the propagator

$$
G_{F}\left(x_{12}\right)=i\left\langle T \varphi\left(x_{1}\right) \varphi\left(x_{2}\right)\right\rangle_{0}=\int_{0}^{\infty} d \lambda \tau(\lambda) \Delta_{\lambda^{2}}\left(x_{12}\right)+\delta_{\eta \eta_{b}} 2 a(\nu-1) \Delta_{0}\left(x_{12}\right) .
$$

If our understanding of [17] is correct, it is claimed there that the Fourier transform

$$
\widehat{G}_{F}(p)=-\int_{0}^{\infty} d \lambda \tau(\lambda) \frac{1}{p^{2}-\lambda^{2}+i \epsilon}-\delta_{\eta \eta_{b}} 2 a(\nu-1) \frac{1}{p^{2}+i \epsilon}
$$

develops complex poles in the variable $p^{2}$. Differently from the flat case (see Eq. (39)), the explicit form of $\widehat{G}_{F}$ for generic $M$ and $\eta$ is quite complicated. Nevertheless, using that $\tau$ is continuous and bounded on $\mathbb{R}_{+}$for any finite $\eta$, we conclude that the $\lambda$-integral in Eq. (66) converges when $p^{2}$ has a non-vanishing imaginary part even for $\epsilon=0$. This excludes the presence of complex poles in $\widehat{G}_{F}$ on the physical sheet of the complex $p^{2}$ plane. Moreover, being a generalized free field, $\varphi$ admits [20] a Fock representation $\mathcal{F}$. 
The subspaces of $\mathcal{F}$ with different particle number are orthogonal and invariant under the Hamiltonian. These features, combined with translation invariance in $\mathbf{M}_{s+1}$, forbid any decay process of $\varphi$. In this sense $\varphi$ is a stable quantum field on the brane.

For $s=3$ the potential $V(r)$ between two static sources on the brane at a distance $r=|\mathbf{x}|$ is

$$
V(r)=\int_{-\infty}^{\infty} d x^{0} G_{F}\left(x^{0}, \mathbf{x}\right)=\int_{0}^{\infty} d \lambda \tau(\lambda) \frac{e^{-\lambda r}}{4 \pi r}+\delta_{\eta \eta_{b}} 2 a(\nu-1) \frac{1}{4 \pi r}
$$

Since $\tau$ is bounded, one has the estimate

$$
V(r) \leq \sup _{\lambda \geq 0}[\tau(\lambda)] \frac{1}{4 \pi r^{2}}+\delta_{\eta \eta_{b}} 2 a(\nu-1) \frac{1}{4 \pi r}
$$

Eq. (68) holds for any $r>0$; for limited regions on $\mathbb{R}_{+}$and selected values of $M$ and $\eta$ one can derive sharper estimates. Suppose for instance we are in the regime when a high peak is present in the spectral function $\tau$. Then, even if at very large $\left(r>>a^{-1}\right)$ and very small $\left(r<<a^{-1}\right)$ distances $V(r)$ has a power-like behavior, there is an intermediate region in which $V(r)$ is well approximated by an Yukawa type potential $\sim \exp (-m r) / r$. The value of $m$ is given by the location of the peak in $\tau$, whereas the limits of validity of the Yukawa approximation are determined by its width.

The cases $s=1,2$ and/or $-[a(s+1) / 2]^{2} \leq M^{2}<0$ can be investigated in analogous way.

\section{Outlook and conclusions}

We studied a quantum scalar field of mass $M$ which propagates in a manifold with boundary representing a $s$-brane. The quantization of this model boils down to the solution of a singular Sturm-Liouville problem with a prescribed boundary condition on the brane, fixed in terms of the parameter $\eta$. Once the bulk 2-point function $\left\langle\Phi\left(x_{1}, y_{1}\right) \Phi\left(x_{2}, y_{2}\right)\right\rangle_{0}$ has been computed, the notion of induced quantum field $\varphi$ on the brane appears naturally, using the limit of $y_{i} \rightarrow 0$. The resulting 2-point function uniquely determines $\varphi$, which turns out to be a generalized free field. Being non-canonical, the dynamics of $\varphi$ cannot be derived from a local Lagrangian defined on the brane. The basic properties of the induced field are captured by the spectral measure of the corresponding Källén-Lehmann representation. The importance of exploring the whole allowed range of $M$ and $\eta$ emerges from our study: both in the flat and in the AdS case, the existence of a boundary state strongly depends on the values of these parameters. We have shown that under certain 
conditions, short range bulk interactions can induce long range forces on the brane. This phenomenon is the consequence of peculiar boundary interactions and to our knowledge has not been observed before. We have seen also that the spectral function $\tau(\lambda)$ in the AdS case has quite complicated behavior. For certain values of $\eta$, it develops a high peak corresponding to excitations with sharply localized mass. This feature is very interesting and deserves further investigation.

Although we have focused in the present paper on a scalar field, our approach applies with slight modifications to fields with higher spin as well. For instance, in the gravitational case the linearized Einstein equations lead to an operator similar to $K$ (see Eq. (43)) with $M=0$. In absence of matter on the brane, Israel's junction conditions impose $\eta=0$. Observing that $\eta_{b}(0)=0$ (see Eq. (45)), the boundary state is present in the spectrum and is responsible for recovering standard gravity on the brane [4]. An interesting problem is the extension of our analysis to the model described in [7]. The boundary state there is not normalizable. Nevertheless, at intermediate scales the induced field reproduces four dimensional gravity. This feature resembles the observed structure in the AdS spectral function $\tau$ for $\eta$ close to $\eta_{b}(M)$.

Finally, is is natural to ask what happens when interactions in the bulk and/or on the brane are turned on. It will be interesting in this respect to perform some perturbative calculation based on the generalized free propagator (65). We are currently working on this issue.

\section{Acknowledgments}

The collaboration of A. Liguori in deriving some of the results of section 2 is kindly acknowledged. We also thank R. Rattazzi for his interest in this work and for useful discussions.

\section{References}

[1] N. Arkani-Hamed, S. Dimopoulos, G. Dvali, hep-ph/9803315 and Phys. Lett. B 429263 (1998); hep-ph/9807344 and Phys. Rev. D 59 (1999) 860;

I. Antoniadis, N. Arkani-Hamed, S. Dimopoulos, G. Dvali, Phys. Lett. B 436 (1998) 257.

[2] G. F. Giudice, R. Rattazzi and J. D. Wells, Nucl. Phys. B 544 (1999) 3.

[3] L. Randall and R. Sundrum, Phys. Rev. Lett. 83 (1999) 3370 and hepph/9905221. 
[4] L. Randall and R. Sundrum, Phys. Rev. Lett. 83 (1999) 4690 and hepth/9906064.

[5] J. Garriga and T. Tanaka, Phys. Rev. Lett. 84 (2000) 2778 and hepth/9911055.

[6] S. B. Giddings, E. Katz and L. Randall, JHEP 0003 (2000) 023.

[7] R. Gregory, V. A. Rubakov and S. M. Sibiryakov, Phys. Rev. Lett. 84 (2000) 592 and hep-th/0002072.

[8] I. Kogan, S. Mouslopoulus, A. Papazoglou, G. Ross and J. Santiago, hepph/9912552; I. Kogan and G. Ross, hep-th/0003074.

[9] C. Csaki, J. Erlich and T. J. Hollowood, hep-th/0003020.

[10] G. Dvali, G. Gabadadze and M. Porrati, hep-th/0002190; hep-th/0003054.

[11] R. Gregory, V. A. Rubakov and S. M. Sibiryakov, hep-th/0003045.

[12] C. Csaki, J. Erlich and T. J. Hollowood, hep-th/0003076.

[13] L. Pilo, R. Rattazzi, A. Zaffaroni, hep-th/0004028.

[14] A. Liguori, M. Mintchev and L. Zhao, Commun. Matyh. Phys. 194 (1998) 569.

[15] A. Liguori and M. Mintchev, Nucl. Phys. B. 522 (1998) 345.

[16] M. Gattobigio, A. Liguori and M. Mintchev, Phys. Lett. B 428 (1998) 143.

[17] S.L. Dubovski, V.A. Rubakov and P.G. Tinyakov, Brane world: disappearing massive matter, hep-th/0006046.

[18] B. Bajc, G. Gabadadze, Phys. Lett. B 474 (2000) 282.

[19] M. Reed and B. Simon, Methods of Modern Mathematical Physics, Vol.2 (Academic Press, New York, 1975).

[20] R.F. Streater and A.S. Wightman, PCT, Spin and Statistics, and All That (Addison-Wesley, Redwood City, 1989).

[21] E.C. Titchmarsh, Eigenfunction Expansions associated with Second-order Differential Equations (Clarendon press, Oxford, 1946). 
[22] M. A. Naimark, Linear Differential Operators, Vol. 1-2 (G.G. Harrap and Co., London, 1967). 